\title{
Segmentation of Clinical Structures from Images of the Human Pelvic Area
}

\author{
Juliana Fernandes Camapum, Alzenir O. Silva, Alan N. Freitas, \\ Hansenclever de F. Bassani, Flávia Mendes O. Freitas \\ Universidade de Brasília, Departamento de Engenharia Elétrica, 70919-970, Brasília, DF, Brasil \\ juliana@ene.unb.br,alzenir@correios.com.br
}

\begin{abstract}
The radiotherapy treatment planning requires the delineation of the therapy structures that will be submitted to the radiation beams. When executed manually, this delineation is a slow process and can result in human errors due to the amount of X-ray Computed Tomography (CT) images that are analyzed in each radiotherapy planning. This process needs precision, minimizing the radiation on healthy areas, close to the target tissues. A new system for automatic segmentation of images of clinical structures is proposed in this work. The algorithm is based on multi-region growing followed by watershed transform. The main contributions are the method of seed pixels selection and predicate of the multi-region growing algorithm and the segmentation results achieved. The system was tested in 400 images and its efficiency was measured by two different statistical methods, correlation and the t-test. The clinical structures of interest are the rectum, bladder and seminal vesicles.
\end{abstract}

\section{Introduction}

Radiotherapy is the apply of ionizing radiation with the objective of destroy or inhibit the growing of cells with abnormal behavior to the organism. This procedure requires the delineation of the therapy structures that will be submitted to the radiation beams. The images of the structures to be treated are acquired from X-ray CT scans and selected for manual or automatic segmentation.

The objective of this work is to help the clinical oncologist in this complex task by providing an accurate and reliable automatic method for image segmentation of therapy relevant structures from the human pelvic area. The regions of interest are rectum, bladder and seminal vesicles.

The segmentation approach proposed in this work is accomplished through the selection of markers called seed pixels. The system needs a certain degree of interaction with the user who selects the amount of markers (in general between three and five). Moreover, he may decide, through visual analyses, if it is necessary to apply morphological filters in order to improve the segmentation. After the pre-processing step, the method consists of applying the watershed in the mosaic image. Beucher [1] uses the gradient and the watershed to get the mosaic image while Bueno [2] calculates the mosaic image through the multi-region growing algorithm. As can be seen in the literature, many researchers have sought the image contour extraction by applying the watershed with other segmentation techniques. Haris [3] proposed an image segmentation technique based on morphological decomposition applying the watershed with a robust algorithm of region merging with dynamic contour. Initially, the technique presented by Eom [4] segments the image trough the watershed transform. Then, the region-merging algorithm is executed in small regions where the borders are overlapped. Hernandez and Barner [5] proposed the implementation of the regionmerging algorithm in the watershed segmentation, applying homogeneity criterion and region integrity.

Our work follows the one started by Bueno [2]. In order to improve the results, we propose a new method of automatic markers detection through the image histogram. This improvement is very important and resulted in precise segmentation of images with poor contrast and acquired from different $\mathrm{CT}$ equipments. In Bueno, the number and gray level of markers were fixed. This restriction limited the algorithm application to her image database and could not be applied to images acquired from other equipments with different contrast and different slices position. 


\section{Methods}

The segmentation model implemented begins with the Multi-Region Growing (MRG) algorithm followed by the watershed transform. Morphological filters may be applied in the pre-processing step to improve the final segmentation. The whole method is based on Mathematical Morphology (MM).

\subsection{Watershed Transform}

In order to understand the watershed transform, we make an analogy between the image function and a topographic relief (Figure 1). The gray level maximum values are the top of the mountains and the regions between these maximum values are the valleys or catchment basins (CB). Each regional minimum is a flooding source and it is associated with a catchment basin. The $\mathrm{CBs}$ are flooded in a constant rate. The watershed lines are the points where two $\mathrm{CB}$ meet, from different flooding sources.

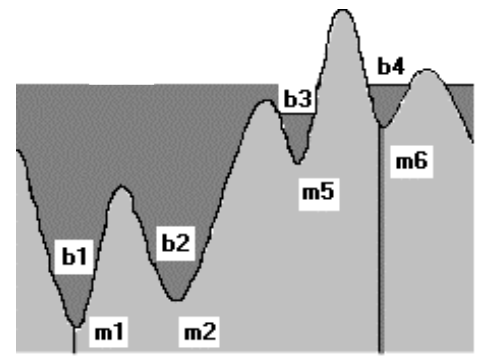

Figure 1. Image function seen as a topographic relief, $\boldsymbol{m}$ are regional minima and $\boldsymbol{b}$ are the valleys (catchment basin).

The most applied watershed algorithms in medical image processing are the ones from Vicente \& Soille [6], which are based on immersion simulations, and the ones from Meyer [7], which are based on hierarchical queues.

The application of the watershed transform in image processing and its solution to many segmentation problems are due to researchers like Digabel [8], Lantuéjoul [9] and Maisonneuve [10].

The watershed transform cannot be applied directly to the image or the image gradient by means of achieving image segmentation because this results in oversegmentation (Figure 2).

Beucher [1] [11] proposed two solutions to this problem based on homotopy modification of the image gradient: one is achieved through morphological reconstruction and the other by creating a mosaic image through the watershed transform. In this paper, we create a mosaic image and calculate the watershed over its gradient.

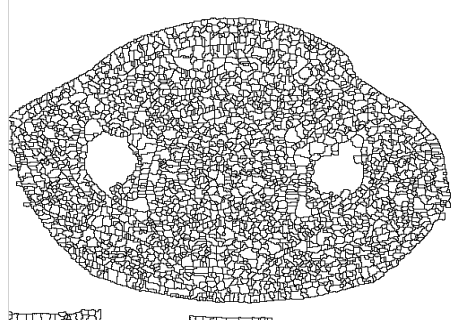

Figure 2. Segmented image after applying the watershed in the gradient image - over-segmentation.

\subsection{Mosaic Image}

The mosaic image is an image composed of homogenous patches. It can be obtained through homotopy modification of the original image. Beucher creates a mosaic image from the watershed transform of the gradient of the original image. This mosaic image is a pre-processing step that will applied in the original image before the segmentation.

We overcome the over-segmentation problem by applying the watershed transform over the mosaic image. In Figure 3, we show the gray level of a line extracted from the mosaic image of a CT image of the pelvic area. In Figure 4, we show the result of applying the multiregion growing in a $\mathrm{CT}$ image of the pelvic area.

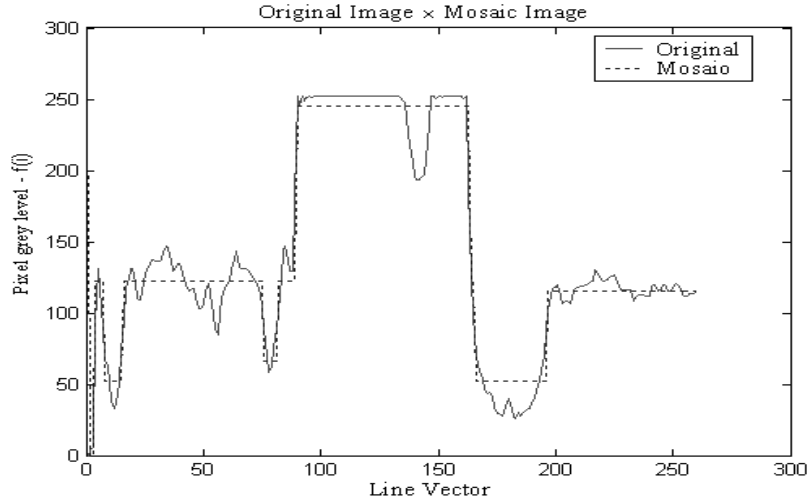

Figure 3. Lines extracted from two images: the input image (full line) and the mosaic image (dotted line).

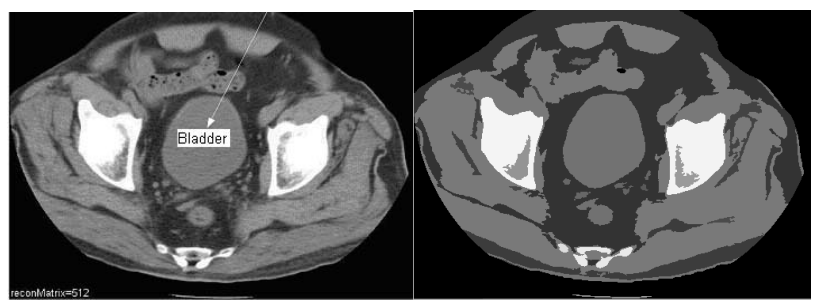

(a)

(b)

Figure 4. (a) Input image (b) mosaic image through multi-region growing. 


\subsection{Multi-Region Growing (MRG)}

In the classic region-growing algorithm [12], the region grows from a single seed and the growing is led through a pre-defined homogeneity criteria. This method can be extended to the MRG where several regions are computed simultaneously.

We decide to implement a new peak detection algorithm based on image histogram. This algorithm provides the seed pixels and the homogeneity criteria to the MRG. Consequently, we do not need to define any parameter. This algorithm was tested in several images of different CT equipments and proved to be totally automatic.

2.3.1. Seed Pixels Selection (markers). The markers selection, known as seed pixels, is computed from the image histogram. The following steps were implemented:

1)Build the image histogram.

2)Determine all the peaks $(\mathrm{P})$ of the histogram.

3)Select, in sequence, some or all the peaks $P$ among the ones that were determined in step 2 , following the rule:

3.1)For each $P$ calculate $g(p i)$

$$
g\left(p_{i}\right)=\text { freq }_{i} \times \text { dist }_{i}^{2}
$$

where $p_{i}$ is the peak $i$ of the histogram, freq $q_{i}$ is the height of the peak and

$$
\text { dist }_{i}=\left\{\begin{array}{l}
1, \text { if } p_{i} \text { is the first peak } \\
\text { horizontal distance to the nearest } \\
\text { selected } p_{i}, \text { otherwise }
\end{array}\right.
$$

The horizontal distance is calculated by the absolute value of the difference between the gray level of the peak been tested and the nearest peak from the ones which have already been selected.

3.2) The peak $p_{i}$, which maximizes the function $\mathrm{g}(\mathrm{pi})$, is selected.

4) All the pixels in the image with gray level of the selected peaks $p_{i}$ from $\mathrm{P}$ are chosen as seed pixels.

Figure 5 shows an example of the steps described above. We consider the graphic as an image histogram. The horizontal values are the gray levels. The vertical values are the frequencies of each gray level. In the second line, $g\left(p_{i}\right)=$ fre $q_{i}$, and $\mathrm{p}_{1}$ is the first selected peak (gray level 2). In the third line, dist $t_{i}$ is the distance of each peak $p_{i}$ from the selected peak $\mathrm{p}_{1}$ and the peak which maximizes $g\left(p_{i}\right)$ is $\mathrm{p}_{4}$. In the end, the selected peaks have gray levels 2, 32 and 19. As a result, all the image pixels with gray levels 2,32 and 19 are classified as seed-pixels. These pixels will initiate the multi-region growing algorithm.

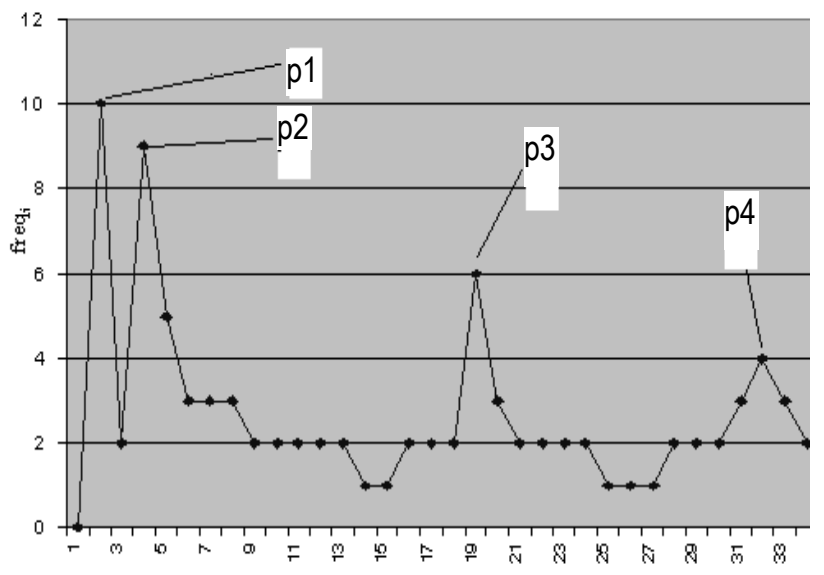

\begin{tabular}{|c|c|c|c|c|}
\hline \hline Selected $\mathrm{p}_{\mathrm{i}}$ & $\mathrm{g}\left(\mathrm{p}_{1}\right)$ & $\mathrm{g}\left(\mathrm{p}_{2}\right)$ & $\mathrm{g}\left(\mathrm{p}_{3}\right)$ & $\mathrm{g}\left(\mathrm{p}_{4}\right)$ \\
\hline- & $10 \times 1$ & $9 \times 1$ & $6 \times 1$ & $4 \times 1$ \\
\hline $\mathrm{p}_{1}$ & - & $9 \times 2^{2}=36$ & $6 \times 17^{2}=1734$ & $4 \times 30^{2}=3600$ \\
\hline $\mathrm{p}_{1}, \mathrm{p}_{4}$ & - & $9 \times 2^{2}=36$ & $6 \times 13^{2}=1014$ & - \\
\hline $\mathrm{p}_{1}, \mathrm{p}_{4}, \mathrm{p}_{3}$ & & & & \\
\hline
\end{tabular}

Figure 5. Selection of histogram peaks as seed pixels.

2.3.2. Homogeneity Criteria. Consider $f(x, y)$ as the gray level of the pixel $(x, y)$. The homogeneity criteria $\left(H_{p i}\right)$ is defined as

$$
H_{p_{i}}(x, y)=\left\{\begin{array}{l}
\text { True, if } v_{i} \leq f(x, y) \leq v_{i+1} \\
\text { False, otherwise }
\end{array}\right.
$$

where the peak $p_{i}$ been tested is located between one valley on the left $v_{\mathrm{i}}$ and one valley on the right $v_{i+1}$. The computation of the gray level of the valleys is presented in Equation 4.

For each peak $p_{i}$ select a gray level $I$ between $p_{i}$ and $p_{i+1}$ that minimize the function $h_{i}(I)$, as defined in the equation below.

$$
\begin{aligned}
& v_{i}=\underset{I}{M I N}\left(h_{i}(I)\right) \\
& h_{i}(I)=\text { freq }_{i} \times\left|I-\frac{\left(I_{i}+I_{i+1}\right)}{2}\right|
\end{aligned}
$$

where: $I$ is a gray level between the gray levels of two selected peaks $p_{i}$ and $p_{i+1}$; freq $q_{i}$ is the frequency of $I$; $\frac{\left(I_{i}+I_{i+1}\right)}{2}$ is the middle value between $p_{i}$ and $p_{i+l}$ and; $\left|I-\frac{\left(I_{i}+I_{i+1}\right)}{2}\right|$ is the distance of $I$ to the middle point between $p_{i}$ and $p_{i+1}$;

The method described above was achieved experimentally and resulted in a very efficient peak detection algorithm. We tested, successfully, in other CT images like mammography images and also compared the 
result with Bueno [2] who provided her image database. The tests proved that the algorithm is independent of the equipment and very reliable.

\section{Results}

The images were presented to radiologists who selected some images for manual segmentation The clinicians criterions were the image contrast and their medical specialization. We have tested an image database of 400 images of X-ray CT scans. The image format is DICOM (Digital Imaging Communications in Medicine) and the dimension is $512 \times 512$.

The software SAPPI-Sistema de Auxílio à Pesquisa em Processamento de Imagens (Assistant Research System for Image Processing) was developed with the compiler Borland $\mathrm{C}++$ Builder $5.0 \AA$, Figure 6. This software has many Mathematical Morphology tools like non-linear filters, the morphological gradient and the watershed transform. The software SAPPI also include the MRG algorithm.
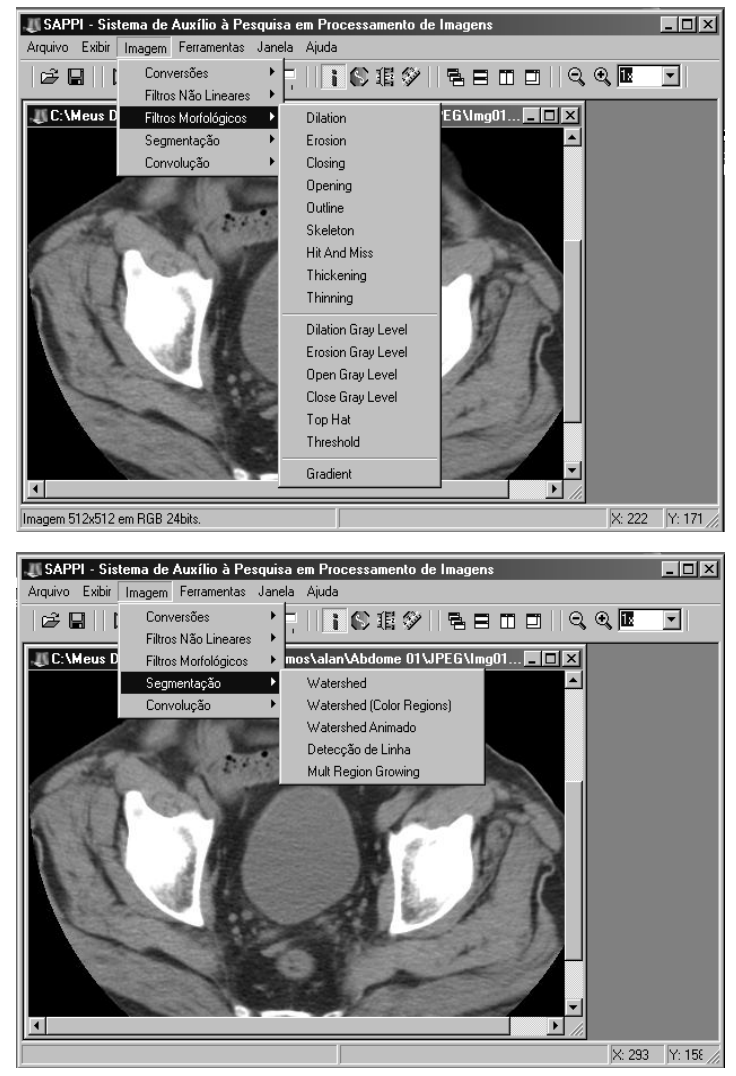

Figure 6. Graphical Interface of the software SAPPI.

The user interact with the SAPPI system during the segmentation process by providing parameters to the morphological filters in the pre-processing step. The user can also define the number of peaks in the MRG algorithm, Figure 7.

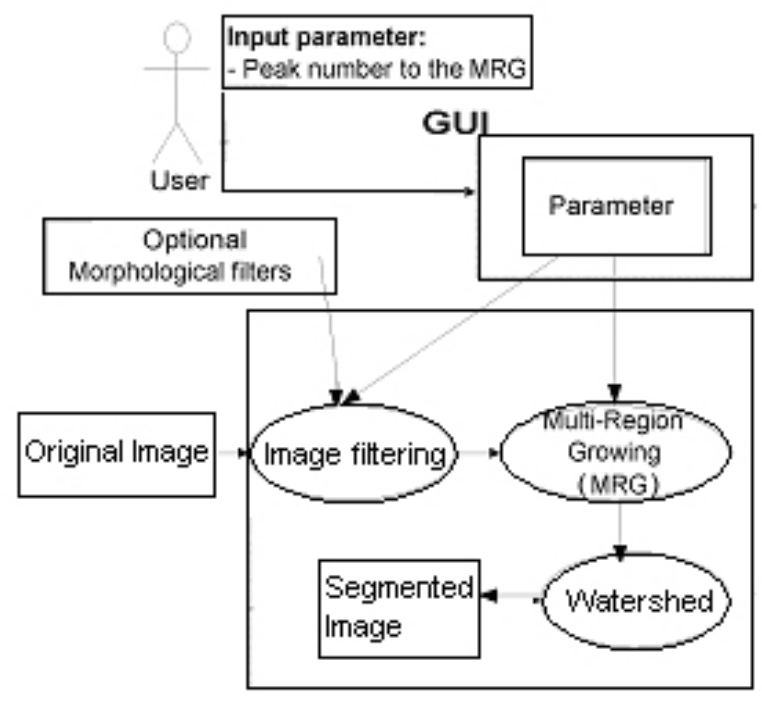

Figure 7. Diagram of the segmentation algorithm.

\subsection{Statistical Measures}

The distance measure between the manual and automatic contour was calculated using the signature of these contours [12]. The signature is represented by the distance $\rho$ of the contour to the center point for different values of angle $\theta$ (Figure 7 and Figure 8).
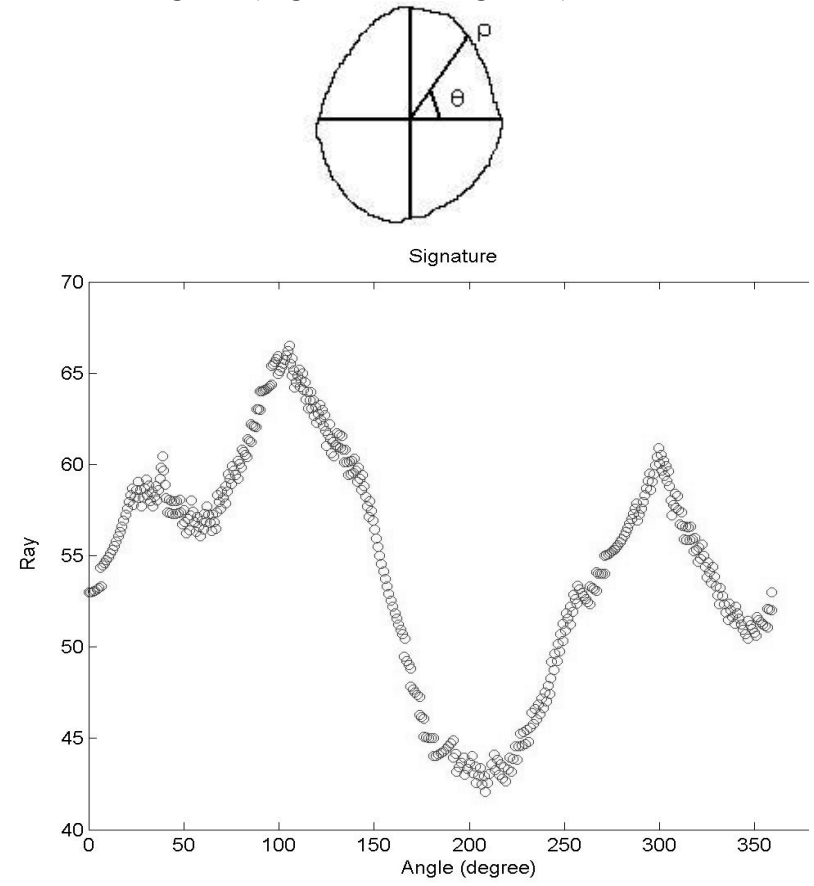

Figure 7. The bladder contour (automatic segmentation) and the signature graphic (ray $\rho \mathrm{x}$ angle $\theta$ ). 


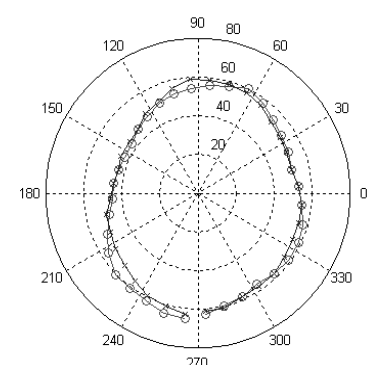

(a)

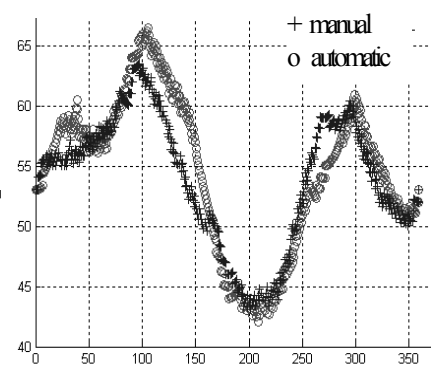

(b)
Figure 8. The comparison between the manual and automatic bladder contour segmentation (a) polar graphic and (b) signature graphic (ray $\rho \mathrm{x}$ angle $\theta$ ).

The statistic analyzed t-test and correlation [13] were undertaken to estimate the error. In the t-test statistic, the critical interval is given by $\left\{(\bar{M}-\bar{A}) \pm\left(t_{\alpha} \times s_{D}\right)\right\}$, where $\alpha$ represents the probability of the null hypothesis be accepted or rejected. If $\alpha$ is small $(\leq 0.05)$, the null hypothesis is rejected. The results depend on the trust level $(100(1-\alpha) \%)$ adopted. The null hypothesis in our tests is "the manual and automatic contours are equal".

$$
\begin{gathered}
t=\frac{|\bar{M}-\bar{A}|}{S_{D}} \\
S_{D}=\sqrt{\frac{\left[\sum_{i=1}^{N_{M}}\left(M_{i}-\bar{M}\right)^{2}\right]+\left[\sum_{i=1}^{N_{A}}\left(A_{i}-\bar{A}\right)^{2}\right]}{N_{M}+N_{A}-2} \times\left(\frac{1}{N_{M}}+\frac{1}{N_{A}}\right)}
\end{gathered}
$$

where $M_{i}$ and $A_{i}$ are the manual and automatic samples of the ray $\rho, \bar{M}$ and $\bar{A}$ are the mean of the manual and automatic samples; $\mathrm{N}_{M}$ and $\mathrm{N}_{\mathrm{A}}$ are the amount of manual and automatic samples and $\left(\mathrm{N}_{\mathrm{M}}+\mathrm{N}_{\mathrm{A}^{-}}\right.$ 2 ) is the degree of freedom of the $t$-test statistic.

The correlation is calculated from Pearson $(r)$ index [13] (Equation (7)). The best result is the one closer to one.

$$
r=\frac{\sum_{i=1}^{N}\left(A_{i}-\bar{A}\right)\left(M_{i}-\bar{M}\right)}{\sqrt{\left(\sum_{i=1}^{N}\left(A_{i}-\bar{A}\right)^{2}\right)\left(\sum_{i=1}^{N}\left(M_{i}-\bar{M}\right)^{2}\right)}}
$$

\subsection{Experimental Results and Performance Analysis}

Some statistical results are shown in Table 1. With degree of freedom of 70 , the best results are achieved with $|t|<1,67$ and the worst results with $|t|>3.43$.

Table 2 presents the segmentation processing time using the following notation:

- PT-Grad - Processing time of the morphological gradient algorithm;

- Peak number (MRG) - Number of peaks defined by the user as a parameter to the MRG;

- Regions number MRG - Number of segmented regions from MRG;

- Regions number after watershed - Number of segmented regions from watershed;

- PT-MRG - MRG processing time;

- $\quad$ PT-Wat - Watershed processing time.

The tests were realized in a P-IV/1.6GHz with 512 Mbytes of RAM. The processing time of all segmentations was very small (in seconds).

Table 1. Statistical results of correlation (r) and t-test (Trust level for null hypothesis - 90\%, 95\% e 99\%.) between manual and automatic segmentation.

\begin{tabular}{|l|c|c|c|c|c|c|c|}
\hline Bladder & $\mathrm{I}-20$ & $\mathrm{I}-22$ & $\mathrm{I}-24$ & $\mathrm{I}-146$ & $\mathrm{I}-211$ & & \\
\hline t-test & $90 \%$ & $90 \%$ & $90 \%$ & $90 \%$ & $90 \%$ & - & - \\
\hline Correlation (r) & 0.9922 & 0.9429 & 0.9381 & 0.9217 & 0.9782 & & \\
\hline Rectum & $\mathrm{I}-28$ & $\mathrm{I}-41$ & $\mathrm{I}-43$ & $\mathrm{I}-48$ & $\mathrm{I}-90$ & $\mathrm{I}-150$ & $\mathrm{I}-211$ \\
\hline t-test & $90 \%$ & $99 \%$ & $99 \%$ & $99 \%$ & $90 \%$ & $90 \%$ & $99 \%$ \\
\hline Correlation (r) & 0.6828 & 0.9727 & 0.8771 & 0.2435 & 0.8634 & 0.9285 & 0.5531 \\
\hline Vesicle & $\mathrm{I}-8$ & $\mathrm{I}-77$ & $\mathrm{I}-79$ & $\mathrm{I}-246$ & & & \\
\hline t-test & $95 \%$ & $90 \%$ & $90 \%$ & & & & \\
\hline Correlation (r) & 0.9479 & 0.9567 & 0.9273 & & & & \\
\hline
\end{tabular}


Table 2. Processing time of the segmentation algorithm.

\begin{tabular}{|c|c|c|c|c|c|c|c|}
\hline Image & $\begin{array}{c}\text { PT-Grad } \\
\text { (sec.) }\end{array}$ & $\begin{array}{c}\text { Peak } \\
\text { number } \\
\text { (MRG) }\end{array}$ & $\begin{array}{c}\text { Regions number } \\
\text { (MRG) }\end{array}$ & $\begin{array}{c}\text { Regions number } \\
\text { after } \\
\text { “watershed" }\end{array}$ & $\begin{array}{c}\text { PT-MRG } \\
\text { (sec.) }\end{array}$ & $\begin{array}{c}\text { PT-Wat } \\
\text { (sec.) }\end{array}$ & $\begin{array}{c}\text { Total Time } \\
\text { (sec.) }\end{array}$ \\
\hline I-20 & 0,993 & 4 & 42 & 98 & 17,950 & 1,015 & 19,958 \\
\hline I-22 & 0,992 & 4 & 52 & 109 & 14,050 & 1,919 & 16,961 \\
\hline I-24 & 0,984 & 4 & 51 & 118 & 15,982 & 1,014 & 17,980 \\
\hline I-146 & 0,085 & 4 & 46 & 98 & 11,058 & 1,014 & 12,157 \\
\hline I-211 & 0,086 & 4 & 49 & 91 & 13,918 & 1,014 & 15,018 \\
\hline I-28 & 0,992 & 4 & 60 & 160 & 18,988 & 1,023 & 21,003 \\
\hline I-41 & 0,993 & 4 & 53 & 133 & 24,983 & 1,022 & 26,998 \\
\hline I-43 & 0,999 & 4 & 42 & 90 & 16,042 & 1,032 & 18,073 \\
\hline
\end{tabular}

Some illustrative examples of the pelvic region segmentation can be seen in Figure 9, Figure 10 and Figure 11. Further results can be seen in [14].

\section{Conclusions}

The statistic measures showed the high accuracy of our segmentation, what make it useful for automatic delineation of organs from medical images.

The multi-region growing algorithm implemented in this work is quite independent of the input image, what is a great advantage of the automatic segmentation algorithm proposed. It was possible to segment the regions of interest (bladder, seminal vesicles and rectum in the case of pelvic images) from medical images with varying contrast and dimension. The seed selection and homogeneity criteria are robust leading to a successful segmentation.

Unfortunately, there are limitations such as when an organ is connected to another organ or when the contrast is very poor for organs in the neighborhood.

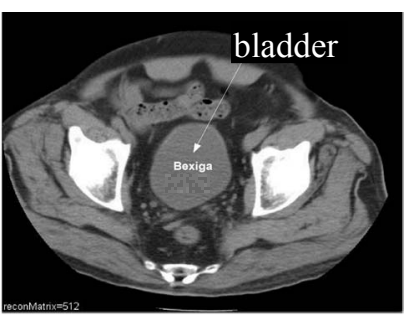

(a)

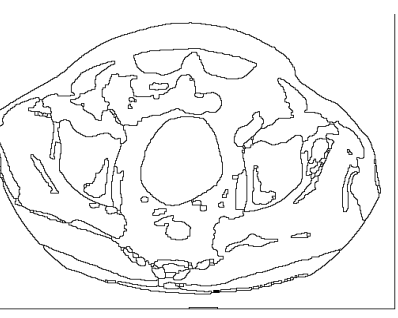

(b)
Figure 9. Bladder segmentation - (a) input image and (b) image after applying our segmentation algorithm.

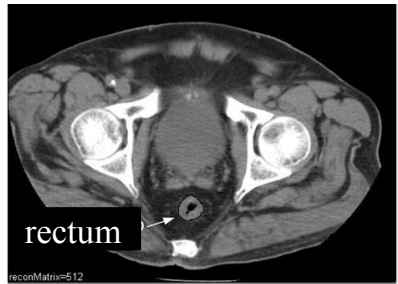

(a)

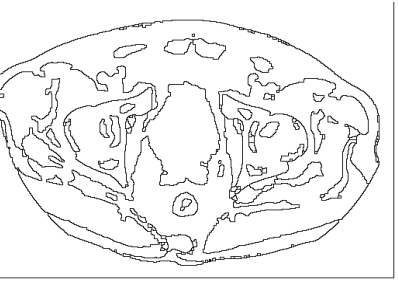

(b)
Figure 10. Rectum: (a) manual segmentation by clinician and (b) automatic result of our segmentation algorithm.

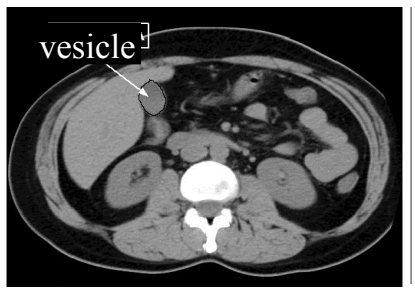

(a)

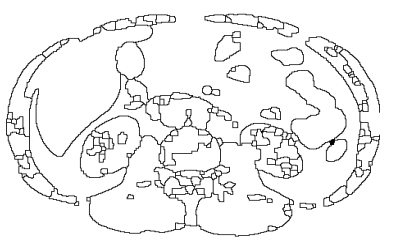

(b)
Figure 11. Seminal vesicles: (a) manual segmentation by clinician and (b) automatic result of our segmentation algorithm

\section{Acknowledgements}

We thank Gloria Bueno for her support in providing part of the algorithm and to the radiologists Dr. Rodrigo Abbdalla de Vasconcelos and Dra. Flávia Mendes who selected the images and delineated the manual contour.

\section{References}

[1] S. Beucher, J. Serra and P. Soille, "Watershed, Hierarchical Segmentation and Waterfall Algorithm", Mathematical Morphology and its Applications to Image Processing, Kluwer Academic Publisher, pp. 69-76, 1994. 
[2] M. G. Bueno, Computer Aided Segmentation of Anatomical Structures in computed Tomographic Images, $\mathrm{PhD}$ Thesis, Coventry University, 1998.

[3] K Haris, S. Efstratiadis and N. Maglaveras, "Hierarchical image segmentation based on contour dynamics", IEEE International Conference on Image Processing, Vol. 1, 2001.

[4] S. Eom, S. Chang and B. Ahn, "Watershed-based region merging using conflicting regions", IEEE International Conference on Image Processing, Vol. 2, 2002.

[5] S. E Hernandez and K. E. Barner, "Joint region merging criteria for watershed-based image segmentation", IEEE International Conference on Image Processing, Vol. 2, 2000.

[6] L. Vincent and P. Soille, "Watersheds in Digital Spaces: An Efficient Algorithm Based on Immersion Simulations", IEEE Transactions on PAMI, Vol. 3, Issue 6, pp. 583-598, 1991

[7] F.Meyer, "Un algorithme optimal de ligne de partage des eaux", 8ème Congrès RFIA, Lyon, France, pp. 847-857, 1991.
[8] H. Digabel and C. Lantuejoul, "Interative algorithms", $2^{\text {nd }}$ European Symp. Quantitative Analysis of Microstructures in Material Science, Biology and Medicine, 1977.

[9] C. Lantuéjoul and S. Beucher, "Use of watershed in contour detection", Int. Workshop Image Processing, Real-Time Edge and Motion Detection/Estimation, 1979.

[10] F. Maisonneuve, "Sur le partage des eaux", Internal Rep CMM, School of Mine, Paris, France, 1982.

[11] S. Beucher, Segmentation d'images et Morphologie Mathématique, PhD thesis, Ecole des Mines de Paris, 1990.

[12] R. C. Gonzalez and R. E. Woods, Digital Image Processing, Editora Prentice Hall, second edition, 2001.

[13] R. C. Clelland, J. S. deCani and F. E. Brown, Basic Statistics with Business Applications, 2th Edition, Canada:John Wiley \& Sons, 1973.

[14] A. O. Silva, Transformada de watershed para a segmentação de imagens médicas da região pélvica, Master dissertation, Universidade de Brasília, Brasília, Brasil, 2003. 